\title{
Use of CBCT Guidance for Tooth Autotransplantation in Children
}

Journal of Dental Research

$1-8$

(C) International \& American Associations

for Dental Research 2019

Article reuse guidelines:

sagepub.com/journals-permissions DOI: |0.| |77/00220345|982870|

journals.sagepub.com/home/jdr

\author{
M. EzEldeen 1,2 $\mathbb{D}$, J. Wyatt ${ }^{2}$, A. Al-Rimawi', W. Coucke', E. Shaheen', \\ I. Lambrichts ${ }^{4}$, G. Willems ${ }^{5}$, C. Politis', and R. Jacobs'
}

\begin{abstract}
Tooth autotransplantation (TAT) offers a viable biological approach to tooth replacement in children and adolescents. The aim of this study was to evaluate the outcome of the cone-beam computed tomographic (CBCT)-guided TAT compared to the conventional TAT protocol and to assess the 3-dimensional (3D) patterns of healing after CBCT-guided TAT (secondary aim). This study included I00 autotransplanted teeth in 88 patients. Each experimental group consisted of 50 transplants in 44 patients ( 31 males and 19 females). The mean (SD) age at the time of surgery was 10.7 (I.I) y for the CBCT-guided group. This was 10.6 (I.3) y for the conventional group. The mean (SD) follow-up period was 4.5 (3.1) y (range, I.I to 10.4 y). Overall survival rate for the CBCT-guided TAT was $92 \%$ with a success rate of $86 \%$ compared to an $84 \%$ survival rate and a $78 \%$ success rate for the conventional group $(P>0.005)$. The following measurements were extracted from the 3D analysis: root hard tissue volume (RV), root length (RL), apical foramen area (AFA), and mean and maximum dentin wall thickness (DWT). Overall, the mean (SD) percentage of tissue change was as follows: RV gain by $65.8 \%$ (34.6\%), RL gain by $37.3 \%$ (31.5\%), AFA reduction by $91.1 \%$ (14.9\%), mean DWT increase by $107.9 \%$ (67.7\%), and maximum DWT increase by $26.5 \%$ (40.1\%). Principal component analysis (PCA) identified the mean DWT, RV, and maximum DWT as the parameters best describing the tissue change after TAT. Cluster analysis applied to the variables chosen by the PCA classified the CBCT group into 4 distinct clusters $(\mathrm{Cl}=37.2 \%, \mathrm{C} 2=17.1 \%, \mathrm{C} 3=28.6 \%, \mathrm{C} 4=17.1 \%)$, revealing different patterns of tissue healing after TAT. The CBCT-guided approach increased the predictability of the treatment. The 3D analysis provided insights into the patterns of healing. CBCT-guided TAT could be adopted as an alternative for the conventional approach. (Clinical trial center and ethical board University Hospitals, KU Leuven: S55287; ClinicalTrials.gov Identifier: NCT02464202)
\end{abstract}

Keywords: CAD, computed tomography, digital imaging/radiology, tooth regeneration/transplantation, periodontal ligament (PDL), clinical outcomes

\section{Introduction}

Tooth autotransplantation (TAT) offers a viable biological approach to tooth replacement in children and adolescents after traumatic dental injuries (TDIs), agenesis, developmental anomalies, or specific orthodontic problems (Czochrowska et al. 2000; Zachrisson et al. 2004; Paulsen et al. 2006). TDIs have a relatively high prevalence $(15.2 \%)$, with children being the most affected (Petti et al. 2018). The treatment options available (e.g., implant placement) are limited by the ongoing dentoalveolar development (Sharma and Vargervik 2006), while orthodontic tooth alignment is challenging unless skeletal anchorage is applied (Kanavakis et al. 2014; Becker et al. 2018). TAT allows for periodontal healing and enables preservation of the alveolar ridge, maintaining the possibility of function and growth (Andreasen, Paulsen, Yu, and Schwartz 1990; Czochrowska et al. 2000; Kallu et al. 2005; Denys et al. 2013). To enhance outcome predictability of the TAT procedure, a low-dose cone-beam computed tomographic (CBCT)guided surgical planning and transfer technique has been developed, involving donor tooth selection and tooth replica fabrication (Shahbazian et al. 2010; Shahbazian et al. 2013).
The primary aim of this study was to evaluate the outcome of the CBCT-guided TAT compared to the conventional TAT protocol, and the secondary aim was to assess the 3-dimensional (3D) patterns of healing after CBCT-guided TAT.

'OMFS IMPATH Research Group, Faculty of Medicine, Department of Imaging and Pathology, KU Leuven and Oral and Maxillofacial Surgery, University Hospitals Leuven, Leuven, Belgium

${ }^{2}$ Department of Oral Health Sciences, KU Leuven and Paediatric Dentistry and Special Dental Care, University Hospitals Leuven, Leuven, Belgium

${ }^{3}$ Certified Freelance Statistician, Heverlee, Heverlee, Belgium ${ }^{4}$ Biomedical Research Institute, Hasselt University, Diepenbeek, Belgium ${ }^{5}$ Department of Oral Health Sciences, KU Leuven and Orthodontics and Dentistry, University Hospitals Leuven, Leuven, Belgium

A supplemental appendix to this article is available online.

\section{Corresponding Author:}

M. EzEldeen, OMFS IMPATH Research Group, Faculty of Medicine, Department of Imaging and Pathology, KU Leuven and Oral and Maxillofacial Surgery, University Hospitals Leuven, Kapucijnenvoer 33, 3000 Leuven, Belgium.

Email: mostafa.ezeldeen@kuleuven.be 


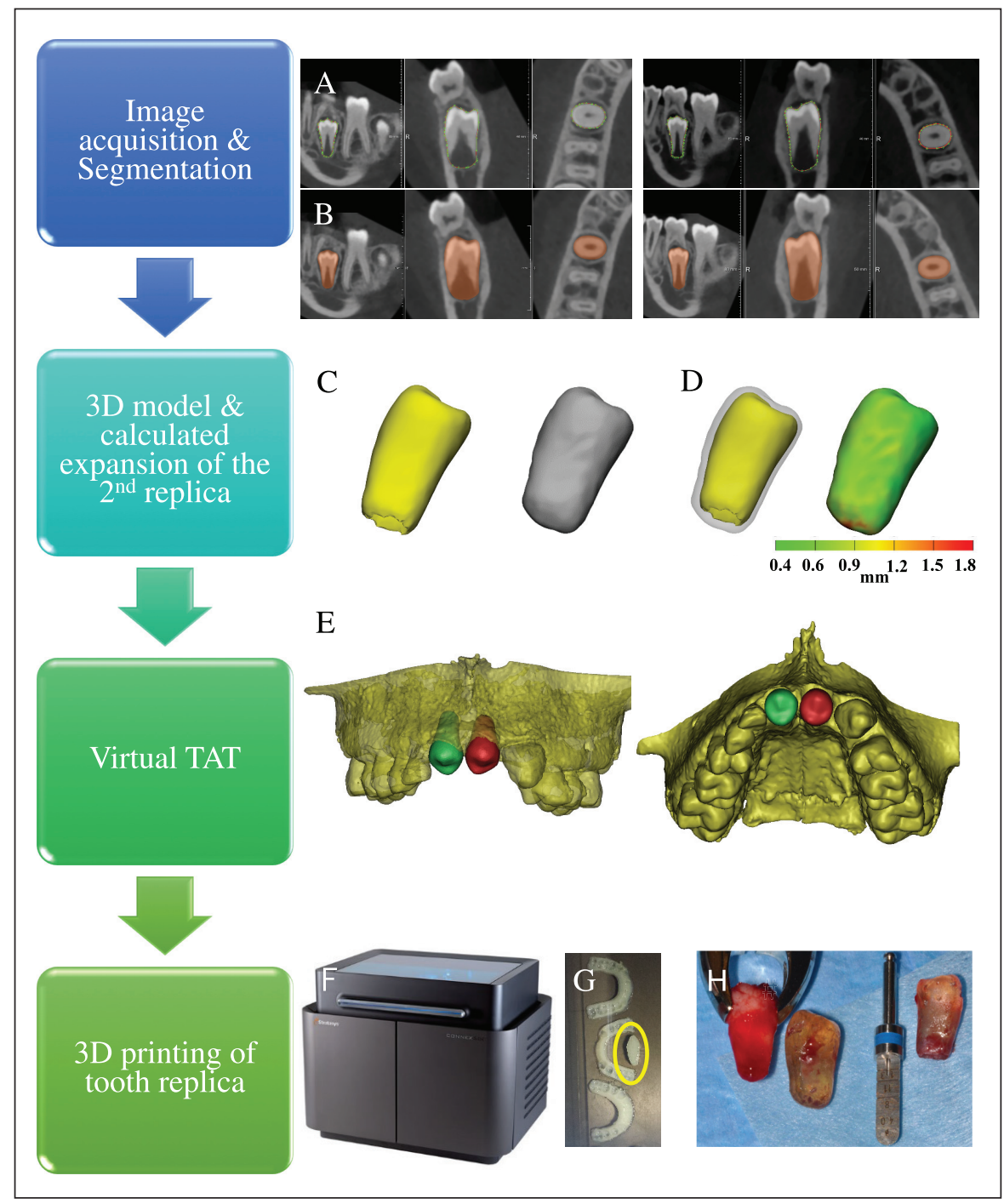

Figure I. Work flow for the preoperative procedure. (A) Cone-beam computed tomographic (CBCT) acquisition; (B) donor tooth segmentation; (C) 3-dimensional (3D) model for the segmented donor tooth, with yellow showing the actual size and gray showing the expanded model accounting for soft tissue and time interval between $\mathrm{CBCT}$ and surgery; (D) second replica expansion ranging between $400 \mu \mathrm{m}$ and I.8 $\mathrm{mm}$ at the apical part; (E) virtual tooth autotransplantation (TAT) for the lower left second premolar (green) to the site of the upper right maxillary central incisor and the lower right second premolar (red) to the site of the upper left maxillary central incisor; (F, G) 3D printing of replica and guides in the in-house 3D printer; and $(\mathbf{H})$ the use of 2 replicas during surgery. consent was obtained. Inclusion criteria were as follows: American Society of Anesthesiologists (ASA) score of $1, \leq 18 \mathrm{y}$ old, and premolar as a donor tooth. The conventional TAT group (control group) consisted of historically matched controls, randomly allocated to each subject at the time of inclusion focusing on similar surgical challenge, age, gender, matching donor tooth with the same stage of root development, and recipient site.

\section{Preoperative Procedure}

A low-dose CBCT scan (see CBCT scanning parameters in the Appendix) (Loubele et al. 2009; EzEldeen et al. 2017) was obtained followed by donor tooth segmentation (Fig. 1A, B) and digital 3D model creation of the donor tooth (Fig. 1C, D) and recipient site (Fig. 1E). A virtual TAT was then performed to determine the surgical feasibility and the best new position for the donor tooth (Fig. 1E). When it was decided for TAT, tooth replica and the surgical guide were 3D-printed and surgery was planned (Fig. 1F-H). The method for CBCTguided surgical planning and tooth replica fabrication was validated and described in detail in previous publications (Shahbazian et al. 2010; Shahbazian et al. 2013).

In the conventional group, 2-dimensional (2D) panoramic radiographs were used to evaluate the recipient site and donor tooth (Denys et al. 2013).

\section{Materials and Methods}

\section{Study Design}

This study was designed as a controlled prospective clinical trial and included 100 autotransplanted teeth in 88 patients. Starting from 2007, all patients referred to the pediatric dentistry department of the University Hospitals of the KU Leuven (Belgium) for TAT and meeting the inclusion criteria were included in the CBCT-guided TAT group (test group). Ethical approval was obtained by the local clinical trial center and ethical board (S55287, University Hospitals, KU Leuven), in compliance with the Helsinki Declaration. This study conformed with STROBE guidelines for human observational studies. Informed

\section{Surgical Procedure}

The procedure for the CBCT-guided group was described in Shahbazian et al. (2013). Briefly, all surgeries took place under general anesthesia by 1 of 2 surgeons. All recipient sites were anesthetized using mepivacaine $3 \%$ without epinephrine (Scandonest; Septodont) to avoid vasoconstriction. The guides and replicas were used to shape the recipient site to match the virtually planned position (Shahbazian et al. 2013). After preparation of the recipient site, the replica was left in place to avoid formation of blood clots (Appendix Fig. 1). The donor tooth was then carefully extracted, avoiding injury to the periodontal ligament membrane, and immediately transplanted to 
the recipient site. The extra-alveolar time of the donor tooth, the number of repositioning attempts, total surgery time, and any surgical complications were recorded. All donor teeth were placed in infraocclusion and initially stabilized with flexible splints, allowing physiological movement. The splinting was carried out using either a flexible orthodontic wire $(0.2 \mathrm{~mm}$ thickness), which was attached using composite to the buccal surfaces of the transplanted and adjacent teeth, or a nonabsorbable nylon suture crossed over the occlusal surface and attached to the tooth occlusal surface using composite (Appendix Fig. 1). All patients were prescribed antibiotics (amoxicillin $50 \mathrm{mg} / \mathrm{kg} / \mathrm{d}$ in 3 doses) for $1 \mathrm{wk}$ postoperatively and instructed to apply chlorhexidine mouthwash locally.

In the conventional group, the surgeries were performed by the same team, according to the procedure described by Andreasen et al. (Andreasen, Paulsen, Yu, Ahlquist, et al. 1990).

\section{Postoperative Clinical and Radiographic Assessment}

In the CBCT-guided group, the subjects were recalled at 1, 4 to 6 , and 12 mo followed by a yearly recall. In the conventional group, clinical and radiographic information was collected from the medical records. At the last recall visit, clinical and radiographic (CBCT imaging) examinations were performed.

Detailed clinical and radiographic examinations were previously described in Shahbazian et al. (2013). Clinical examination included the following parameters: sensibility, color, mobility, tenderness to percussion, percussion tone, probing pocket depth, and gingival status of the transplanted teeth. Radiographic examination included periapical radiographs at recall visits and $\mathrm{CBCT}$ at 1,2 , and $\geq 5 \mathrm{y}$ postoperatively. Radiographs were used to evaluate signs of pulp canal obliteration, status of the periradicular area, root length, root crown ratio, periodontal ligament space (PDL), and root resorption.

A TAT was considered successful when the transplanted tooth had normal clinical and radiographic findings with the absence of ankylosis, progressive root resorption or infection, the presence of root canal obliteration, normal mobility and normal gingival contour, attachment level, and pocket depth. The tooth survival was defined as the transplanted tooth still being present at the follow-up period with or without fulfilling the success criteria.

TAT was considered a failure when the transplanted tooth was already extracted or when there was replacement resorption (RR), hypermobility, or progressive root resorption due to infection.

\section{D Analysis of TAT Outcome}

For the CBCT-guided group, CBCT scans were imported into MeVisLab (MeVis Medical Solutions AG). The transplanted teeth (pre- and postoperatively at last follow-up) were then segmented using a dedicated tool developed in MeVisLab and validated for accurate tooth/root canal space segmentation as described (EzEldeen et al. 2015). Briefly, the imaging analysis tool applies semi-interactive livewire boundary extraction (Barrett and Mortensen 1997) to create a set of orthogonal contours, followed by a variational interpolation algorithm that reconstructs the surface of an object with energy-minimizing, smooth, and implicit functions (Heckel et al. 2011). At this point, the apical foramen area (AFA) at the most apical axial slice was measured and the root canal space was subtracted from the segmented image of the tooth pre- and postoperatively. For each tooth, the segmented hard tissue preoperative image was then spatially aligned to the segmented postoperative image using the dentin-enamel and the cement-enamel junctions as alignment landmarks and maximization of mutual information as a registration metric (Maes et al. 1997). The 3D triangle-based surface of the hard tissue was reconstructed. The root hard tissue volume (RV) and its length (RL) were calculated measuring from the cement-enamel junction (CEJ) to the apex pre- and postoperatively. Morphological quantification was performed in 3matic (Materialise). The root hard tissue was analyzed to record the mean and maximum dentin wall thickness (DWT) pre- and postoperatively and then expressed as a color-coded map showing the calculated minimal distances from the canal surface to the external root surface. The measurements were then normalized and expressed as the percentage of change related to the preoperative form.

\section{Statistical Analysis}

Statistical modeling and analysis were performed in S-plus for Linux 8.0 (Tibco Software) by a certified statistician. Graphs were plotted using the statistical software package GraphPad Prism 7.00 (GraphPad Software). The overall survival, PDL healing, and pulp healing of the CBCT-guided and the conventional groups were modeled and compared using Frailty regression models to account for the dependence in the data. Frailty regression models were fit to the $\mathrm{CBCT}$ group to examine if any factors had an influence on the survival of TAT (see variable regression models in the Appendix). A principal component analysis (PCA) was performed to determine the parameters best describing the total variability in the 3D tissue change after TAT. A cluster analysis (elbow method), using the parameters chosen by the PCA, was then applied to classify the study sample based on the pattern of tissue change.

\section{Results}

\section{Study Sample}

Figure 2A presents the distribution of patients in both groups. Each experimental group consisted of 50 transplants in 44 patients (31 males and 19 females). The mean (SD) age at the time of surgery was 10.7 (1.1) y (range, 8 to $13 \mathrm{y}$ ) for the CBCT-guided group compared to 10.6 (1.3) y (range, 8 to $13 \mathrm{y}$ ) for the conventional group. The mean (SD) follow-up period for both groups was 4.5 (3.1) y (range, 1.1 to $10.4 \mathrm{y}$ ) (25th to 75 th percentile range, 1.7 to $7.4 \mathrm{y}$ ). 


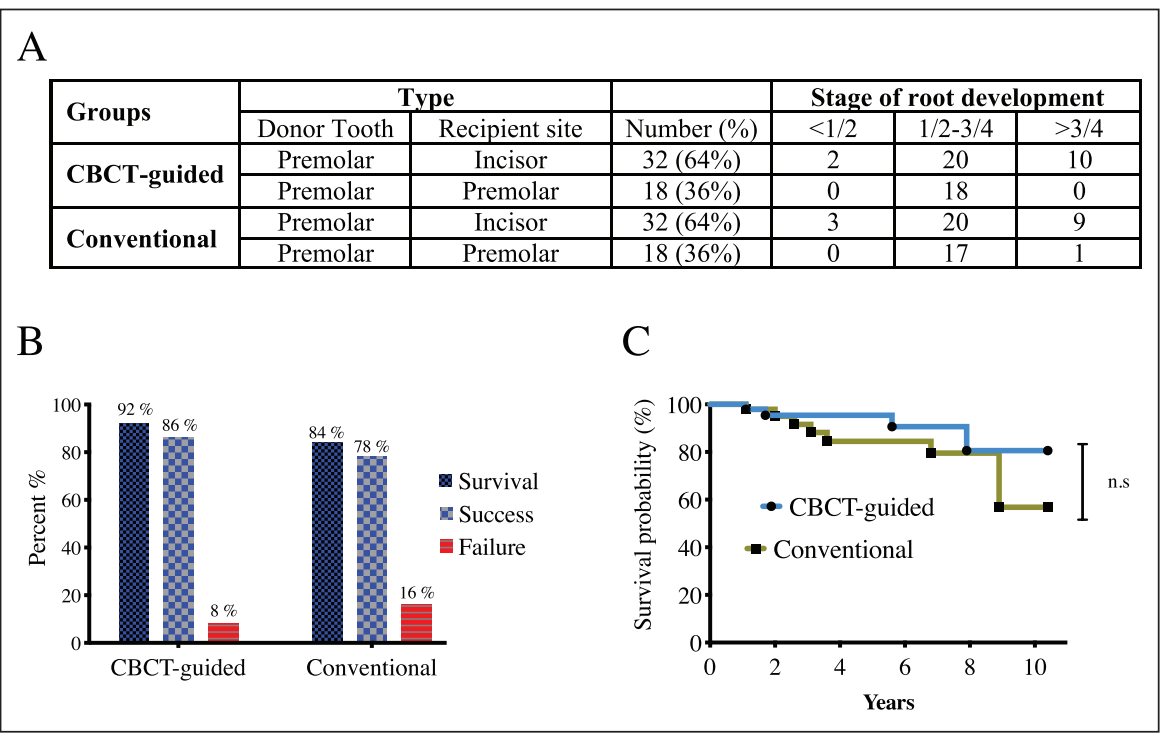

Figure 2. Outcomes of cone-beam computed tomographic (CBCT)-guided versus conventional tooth autotransplantation (TAT). (A) Distribution of patients in CBCT and conventional groups; (B) overall survival, success, and failure distribution, and (C) TAT survival probability over time (baseline to $10 \mathrm{y}$ ).

\section{D Analysis of \\ TAT Outcome and Radiographic Patterns of Healing}

The following measurements were extracted from the 3D analysis: RV, RL, AFA, and mean and maximum DWT. Overall, the mean (SD) percentage of tissue change was RV gain by $65.8 \%$ (34.6\%), RL gain by $37.3 \%$ (31.5\%), AFA reduction by 91.1\% (14.9\%), mean DWT increase by $107.9 \%$ (67.7\%), and maximum DWT change by $26.5 \%$ (40.1\%).

PCA showed that a total of $87.5 \%$ of the variability in tissue change after TAT could be explained using a $3 \mathrm{D}$ space consisting of 3 components (Appendix Fig. 2A). PCA revealed that the main descriptor for tooth hard tissue change postoperatively was the change in the mean DWT (explaining $57.8 \%$ of the variability)

\section{CBCT-Guided versus Conventional TAT}

Overall survival rate for the CBCT-guided TAT was $92 \%$ and success rate was $86 \%$ compared to an $84 \%$ survival rate and a $78 \%$ success rate for the conventional group (Fig. 2B). Differences between both groups were not statistically significant $(P>0.05)$. The PDL and pulp-healing rates for the CBCT group were $86 \%$ and $92 \%$, respectively, compared to $82 \%$ and $88 \%$ for the conventional group. Differences between both groups were not statistically significant $(P>0.05)$. Figure 3 presents an example for the long-term outcome after TAT. The use of the tooth replica reduced the number of repeated attempts of repositioning the donor tooth to 0 to 3 attempts for the CBCT group compared to 4 to 7 attempts for the conventional group. The extra-alveolar time was reduced to $<1 \mathrm{~min}$ for the $\mathrm{CBCT}$ group compared to 3 to $10 \mathrm{~min}$ for the conventional group. Moreover, the total surgery time was reduced from 40 to $90 \mathrm{~min}$ for the conventional group to 30 to $45 \mathrm{~min}$ for the CBCT group.

Reasons for failures in the CBCT group were ankylosis accompanied by RR (3 TATs) or untreated caries leading to periapical infection (1 TAT), while for the conventional group, reasons were inflammatory root resorption (5 TATs), ankylosis with RR (2 TATs), or external cervical root resorption (1 TAT).

The regression model constructed with the pre- and intraoperative variables did not reveal any variables with significant relation to the outcome. Only gender appeared to have a close relationship to the overall survival $(P=0.074)$, with males more likely to have a higher TAT survival rate than females.
(Appendix Fig. 2A, B). This was followed by the change in RV $(17.1 \%)$ and the change in maximum DWT (12.6\%) (Appendix Fig. 2A, C). Moreover, factor (component) analysis (Varimax rotation method) showed that the change in mean DWT is strongly correlated to the reduction in AFA (first component) and the change in $\mathrm{RV}$ is strongly correlated to the change in $\mathrm{RL}$ (second component). AFA and RL had less power explaining the first and second components, and thus they were excluded from further analysis (Appendix Fig. 2B, C). Interestingly, the change in the maximum DWT had a strong relation to the third component but with a low relation to the first and second components (Appendix Fig. 2C). Frailty regression model revealed a significant relationship between the change in mean DWT and the time interval until PDL and pulp healing $(P<0.0001)$. It was not possible to examine the relationship between the change in mean DWT and the overall success and survival because of the censored nature of the data.

Cluster analysis applied to the variables chosen by the PCA revealed that the $\mathrm{CBCT}$ group could be classified into 4 distinct clusters $(\mathrm{C} 1=37.2 \%, \mathrm{C} 2=17.1 \%, \mathrm{C} 3=28.6 \%, \mathrm{C} 4=$ $17.1 \%$ ) (Fig. 4). Analyzing cluster characteristics showed no differences preoperatively (Fig. 5A, C, E) but different patterns of tissue change postoperatively (Fig. 5B, D, F). The change in mean (SD) DWT was the parameter mainly distinguishing between the clusters as follows: $\mathrm{C} 1$ increase by $36 \%(22 \%), \mathrm{C} 2$ increase by $188 \%(34 \%), \mathrm{C} 3$ increase by $100 \%(30 \%)$, and C4 increase by $166 \%$ (20\%) (Fig. 5B). While clusters 2 and 4 showed overlap (Fig. 4B), they could be differentiated using 
the change in the maximum DWT (Fig. 4D), showing a mean (SD) increase by $25 \%(19 \%)$ with $\mathrm{C} 2$ and 97\% (21\%) with C4. Examining the influence of the mean (SD) followup period showed only significant differences $(P<0.05)$ between 2.6 (2.3) y for $\mathrm{C} 1$ and $7.5(0.8)$ y for $\mathrm{C} 4$ (Fig. 5D).

\section{Discussion}

Application of computer-aided surgical planning and transfer technique combined with 3D printing of tooth replica and surgical templates for TAT (CBCT guided) aims to examine the surgical feasibility, plan the ideal position for the donor tooth in relation to the neighboring teeth and recipient site, and enhance esthetics and function (Shahbazian et al. 2013). The use of tooth replica intends to reduce the extra-alveolar time and provide a passive adaptability for the donor tooth through bone contouring, leading to preserving the periodontal membrane and pulp vitality, as well as reducing the risk of necrosis and resorption (Keightley et al. 2010; Shahbazian et al. 2010; Shahbazian et al. 2013; Jang et al. 2016). The current study provides results on an intermediateto long-term TAT follow-up comparing CBCT-guided to the conventional approach, focusing on immature premolars in children.

Available data in the literature on conventional TAT reflect comparable long-term survival rates (Akhlef et al. 2018). Kafourou et al. (2017) reported overall survival and success rates of $94.4 \%$ and $85.4 \%$, respectively, for conventional TAT in children and adolescents after a mean (SD) follow-up period of $2.6(1.8) \mathrm{y}$. In the current study, CBCT-guided TAT showed slightly higher success and survival rates when compared to the conventional approach (Fig. 2B). Generally, the differences between the 2 groups were not statistically significant. Nevertheless, such differences are significant for the individual patient. Looking at the survival curves (Fig. 2C), it can be noticed that at longer follow-up periods, the survival probability for the CBCT group stays well above $80 \%$, while it drops below $60 \%$ for the conventional group.

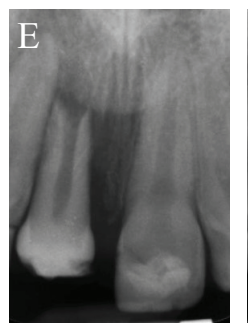

$\mathrm{L}$

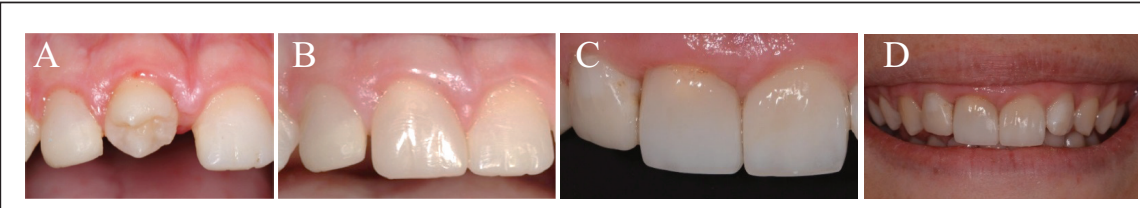

1 year
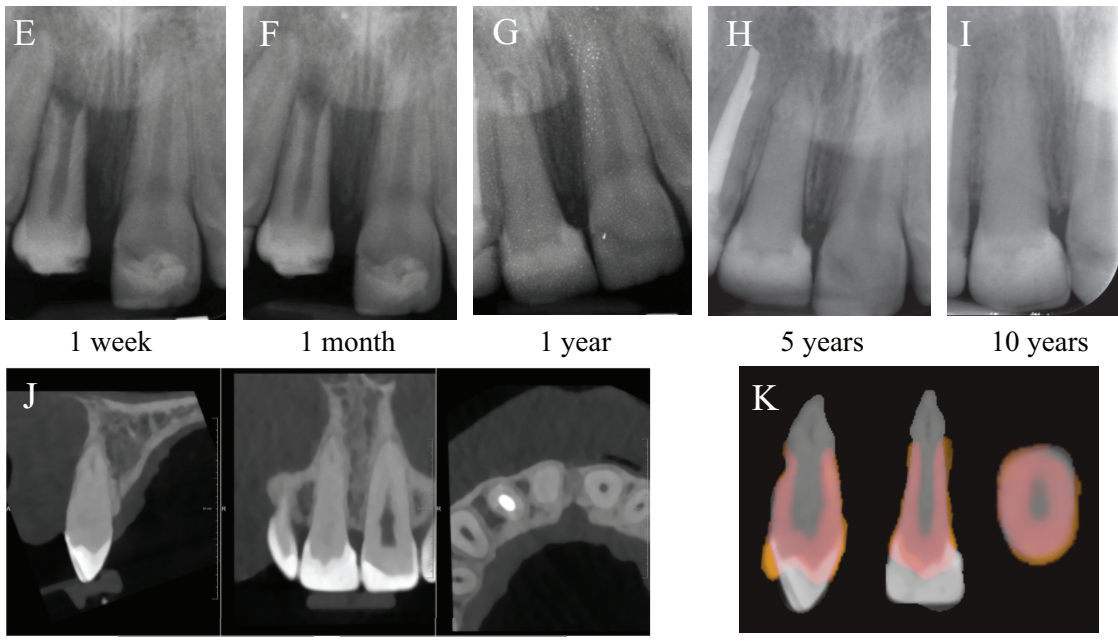

CBCT at 10 years post-op

Hard tissue thickness pre and post-op
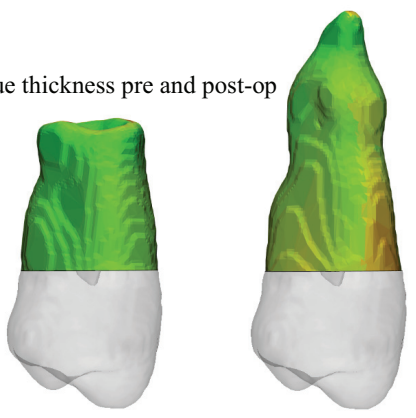

M

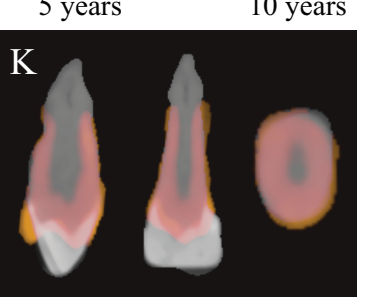

Pre-op vs. Post-op

Figure 3. Long-term outcome after tooth autotransplantation (TAT), lower left second mandibular premolar to the site of the maxillary right central incisor. Clinical and esthetic outcome at (A) I mo, (B) I y, and (C, D) 10 y. (E-I) Radiographic follow-up and successful outcome at 10 y, (J, K) conebeam computed tomography $(C B C T)$ at 10 y postoperatively, and (L) 3-dimensional analysis of the hard tissue thickness. Preoperatively: maximum dentin thickness $=4.1 \mathrm{~mm}$ (mean $[\mathrm{SD}]=1.7[0.5]$ $\mathrm{mm}$; postoperatively: maximum dentin thickness $=5.9 \mathrm{~mm}$ (mean $[\mathrm{SD}]=3.1$ [ 1.4$] \mathrm{mm})$. (M) Surface distance mask representing tissue change postoperatively.

Interestingly, not only the number of failures was halved (Fig. 2B), but also the types of failure were different. The main reason for failure for the $\mathrm{CBCT}$ group was ankylosis accompanied by RR, while this was of an inflammatory nature for the conventional approach. Repeated repositioning attempts and extended extra-alveolar times will damage the periodontal membrane and reduce the chances of pulp revascularization (Andreasen, Paulsen, Yu, Bayer, and Schwartz 1990; Andreasen, Paulsen, Yu, and Schwartz 1990), thus explaining the inflammatory nature of complications in the conventional 


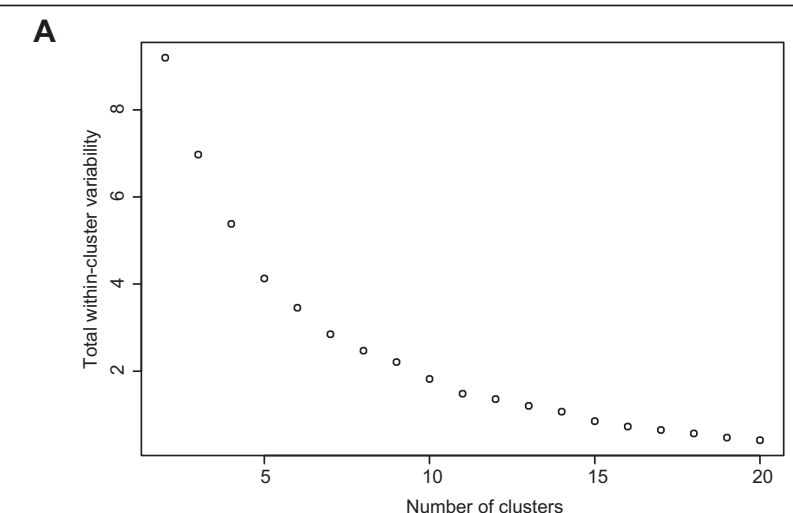

B

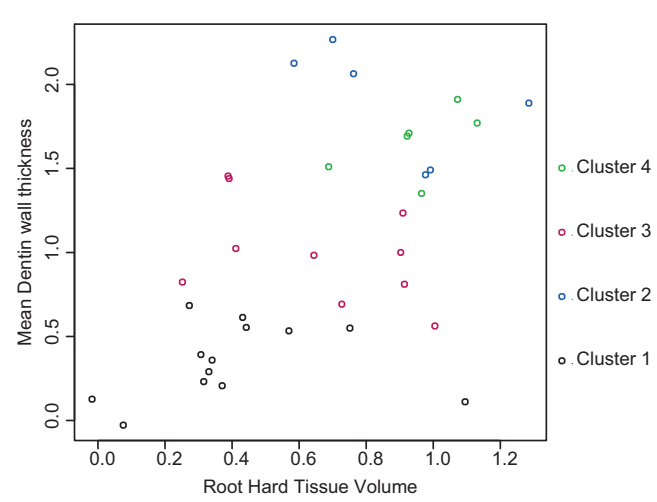

C

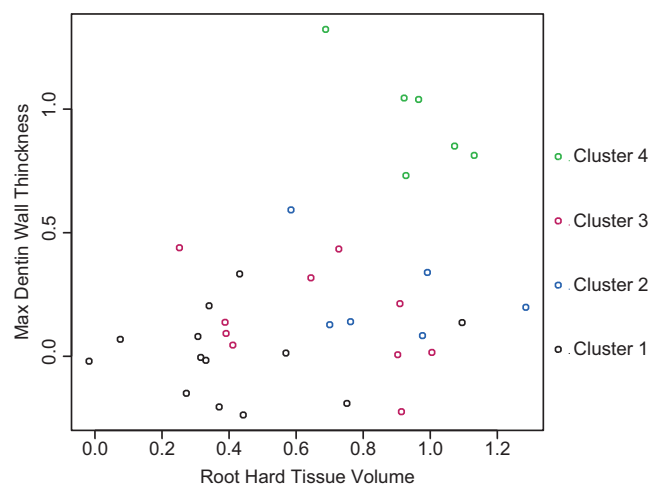

D

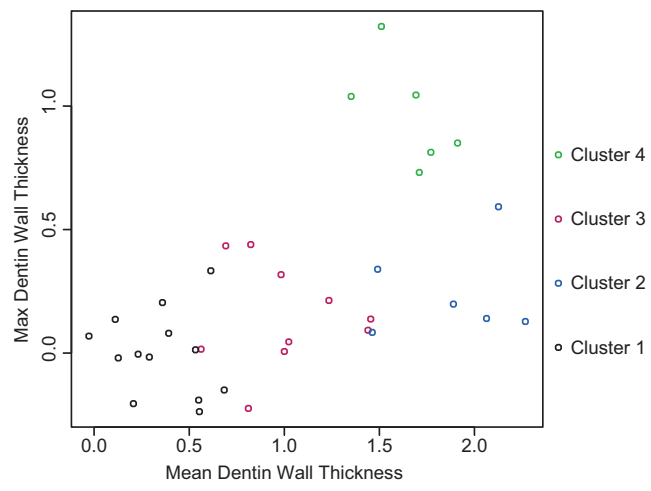

Figure 4. Cluster analysis. (A) For obtaining the optimal number of clusters, the elbow method was used, whereby the total within-cluster variability was plotted versus the number of clusters. The steepness of the plotted line changed at 5 clusters; thus, initially a cluster analysis with 5 clusters was performed and then optimized to 4 clusters. (B, C) Mean group. On the other hand, the ankylosis followed by RR in the $\mathrm{CBCT}$ group might be due to a tight fit of the donor tooth at the recipient site. One possible explanation is that the donor tooth will be larger than the replica when the time interval between the CBCT acquisition and the surgery exceeds 2 to $4 \mathrm{mo}$. This complication can be avoided by accounting for the root growth, apical papilla, and PDL membrane by creating a second replica with calculated root expansion (Fig. 1C, D).

Root canal obliteration and continued root development after TAT of immature teeth are signs of pulp revitalization and treatment success (Andreasen, Paulsen, Yu, and Bayer 1990; Andreasen, Paulsen, Yu, Bayer, and Schwartz, 1990; Czochrowska et al. 2002; Tsukiboshi 2002). This study applied a novel method based on 3D imaging to analyze the pattern of hard tissue formation and subsequent healing after TAT. Our results suggest 4 radiographic patterns of healing after TAT of immature premolars. These patterns have a 4-dimensional nature: change in mean DWT, RV, and maximum DWT influenced by the time factor. Change in mean DWT and RV appeared to plateau after the first 3 to $4 \mathrm{y}$, while the change in the maximum DWT appeared to have a gradual increase during the follow-up period (Fig. 5B, D, F). One possible interpretation is that the change in maximum DWT describes the slower tissue remodeling on the external root surface at the tooth root, PDL-bone interface. On the other hand, change in mean DWT and RV describes the faster tissue deposition on the internal root surface and overall root maturation. This information would assist the clinicians during the follow-up in understating what is observed in the radiographic examination. Future work will focus on expanding this concept based on studies with larger sample size.

With the rapid advances in stem cell biology, biomaterials, biofabrication, and tissue engineering strategies, tooth regeneration seems to be possible sooner than expected (Wei et al. 2013; Obregon et al. 2015; Hilkens et al. 2017; Lambrichts et al. 2017; Smith et al. 2018). TAT represents a unique biological treatment option that offers not only a solution for tooth loss in children and adolescents but also insights for future bioengineered tooth transplants. The design of a bioengineered tooth root can apply lessons regarding scaffold dimensions, the most suitable developmental stage for transplantation, and expected healing patterns. Moreover, TAT appears to be a more predictable approach with the aid of 3D planning and 3D printing of tooth replica and surgical guides.

It is fair to mention that this study has its limitations. It was not possible to design the study as a randomized clinical trial and allocate new patients in the prospective arm to the conventional group for ethical reasons. Moreover, 3D imaging was not available for the conventional group preoperatively; therefore, a direct comparison of the healing patterns between the $\mathrm{CBCT}$ and conventional groups was not possible. The number

dentin wall thickness (DWT) was the variable differentiating the groups the best into horizontal bands, with clusters 2 and 4 showing a strong overlap. (D) Clusters 2 and 4 can be separated using the maximum DWT. 
of failures was limited; therefore, it was not possible to draw conclusions regarding the risk factors or prognostic variables. However, the data presented in this study are unique. This study has provided an intermediate- to long-term followup on CBCT-guided TAT compared to the conventional approach. Moreover, meaningful parameters were identified for the $3 \mathrm{D}$ analysis and reporting of the treatment outcome, in addition to suggesting 4 patterns for the radiographic healing after TAT. Furthermore, the 3D parameters identified can be extended to studying the regenerative endodontic procedures (REPs) (EzEldeen et al. 2015; Meschi et al. 2018) to obtain insights into the patterns of healing and determinants of success. Future studies should focus on gaining a deeper understanding for the biology-based procedures such as TAT and REPs to preserve the natural dentition and maximize the benefits for the patient.

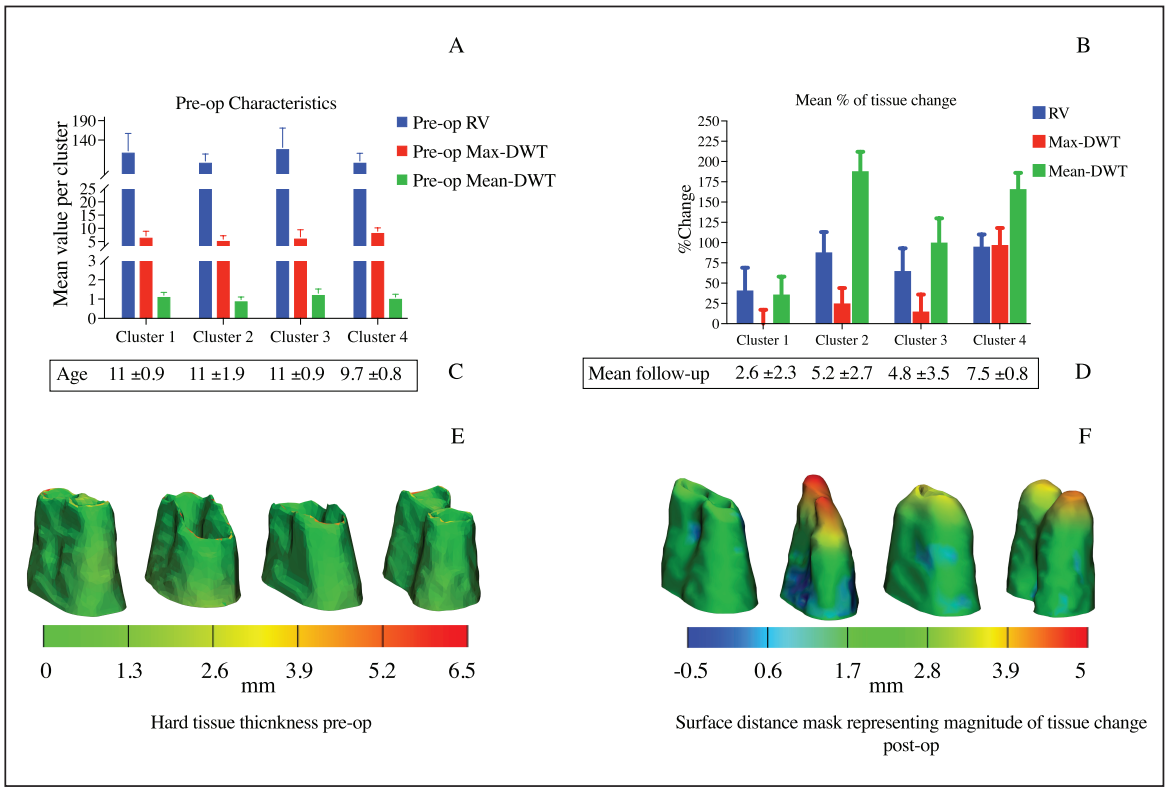

Figure 5. Different patterns of healing after tooth autotransplantation (TAT). (A) The preoperative characteristics for the 4 clusters identified showing homogeneous values for root hard tissue volume (RV), maximum dentin wall thickness (DWT), and mean DWT; (B) different patterns of tissue change between the 4 clusters postoperatively; (C) similar mean age between the clusters preoperatively; (D) mean follow-up period for the 4 clusters; (E) color-coded map for the hard tissue thickness, for I example from each cluster, showing the similarities; and (F) surface distance mask representing the magnitude of tissue change postoperatively, clearly showing the different patterns of healing and remodeling after TAT.

\section{Conclusion}

TAT is a viable option for permanent tooth replacement in children and adolescents. The CBCT-guided approach simplified the surgery and increased the predictability of the treatment. The 3D analysis provided insights into the patterns of healing. CBCT-guided TAT could be adopted as an alternative for the conventional approach.

\section{Author Contributions}

M. EzEldeen, contributed to conception, design, data acquisition, analysis and interpretation, drafted and critically revised the manuscript; J. Wyatt, R. Jacobs, contributed to conception, design, data acquisition, and interpretation, drafted and critically revised the manuscript; A. Al-Rimawi, contributed to data acquisition, and analysis, drafted the manuscript; W. Coucke, contributed to conception, data analysis, and interpretation, drafted the manuscript; E. Shaheen, contributed to data acquisition and interpretation, critically revised the manuscript; I. Lambrichts, G. Willems, contributed to conception and design, critically revised the manuscript; $\mathrm{C}$. Politis, contributed to conception, critically revised the manuscript. All authors gave final approval and agree to be accountable for all aspects of the work.

\section{Acknowledgments}

Supported by FWO Vlanderen (Fonds Wetenschapelijk Onderzoek) (grant no. G089213N). The authors declare no potential conflicts of interest with respect to the authorship and/or publication of this article.

\section{ORCID iD}

M. EzEldeen (iD https://orcid.org/0000-0002-9992-1138

\section{References}

Akhlef Y, Schwartz O, Andreasen JO, Jensen SS. 2018. Autotransplantation of teeth to the anterior maxilla: a systematic review of survival and success, aesthetic presentation and patient-reported outcome. Dent Traumatol. 34(1):20-27.

Andreasen JO, Paulsen HU, Yu Z, Ahlquist R, Bayer T, Schwartz O. 1990. A long-term study of 370 autotransplanted premolars: Part I. Surgical procedures and standardized techniques for monitoring healing. Eur J Orthod. 12(1):3-13.

Andreasen JO, Paulsen HU, Yu Z, Bayer T. 1990. A long-term study of 370 autotransplanted premolars: Part IV. Root development subsequent to transplantation. Eur J Orthod. 12(1):38-50.

Andreasen JO, Paulsen HU, Yu Z, Bayer T, Schwartz O. 1990. A long-term study of 370 autotransplanted premolars. Part ii. Tooth survival and pulp healing subsequent to transplantation. Eur J Orthod. 12(1):14-24.

Andreasen JO, Paulsen HU, Yu Z, Schwartz O. 1990. A long-term study of 370 autotransplanted premolars. Part iii. Periodontal healing subsequent to transplantation. Eur J Orthod. 12(1):25-37.

Barrett WA, Mortensen EN. 1997. Interactive live-wire boundary extraction. MedImage Anal. 1(4):331-341.

Becker K, Wilmes B, Grandjean C, Vasudavan S, Drescher D. 2018. Skeletally anchored mesialization of molars using digitized casts and two surfacematching approaches: analysis of treatment effects. J Orofac Orthop. 79(1):11-18.

Czochrowska EM, Stenvik A, Album B, Zachrisson BU. 2000. Autotransplantation of premolars to replace maxillary incisors: a comparison with natural incisors. Am J Orthod Dentofacial Orthop. 118(6):592600 . 
Czochrowska EM, Stenvik A, Bjercke B, Zachrisson BU. 2002. Outcome of tooth transplantation: survival and success rates $17-41$ years posttreatment. Am J Orthod Dentofacial Orthop. 121(2):110-119.

Denys D, Shahbazian M, Jacobs R, Laenen A, Wyatt J, Vinckier F, Willems G. 2013. Importance of root development in autotransplantations: a retrospective study of 137 teeth with a follow-up period varying from 1 week to 14 years. Eur J Orthod. 35(5):680-688.

EzEldeen M, Stratis A, Coucke W, Codari M, Politis C, Jacobs R. 2017. As low dose as sufficient quality: optimization of cone-beam computed tomographic scanning protocol for tooth autotransplantation planning and follow-up in children. J Endod. 43(2):210-217.

EzEldeen M, Van Gorp G, Van Dessel J, Vandermeulen D, Jacobs R. 2015. 3-Dimensional analysis of regenerative endodontic treatment outcome. J Endod. 41(3):317-324.

Heckel F, Konrad O, Karl Hahn H, Peitgen H-O. 2011. Interactive 3D medical image segmentation with energy-minimizing implicit functions. Comp Graph. 35(2):275-287.

Hilkens P, Bronckaers A, Ratajczak J, Gervois P, Wolfs E, Lambrichts I. 2017. The angiogenic potential of DPSCs and SCAPs in an in vivo model of dental pulp regeneration. Stem Cells Int. 2017:2582080.

Jang Y, Choi YJ, Lee SJ, Roh BD, Park SH, Kim E. 2016. Prognostic factors for clinical outcomes in autotransplantation of teeth with complete root formation: survival analysis for up to 12 years. J Endod. 42(2):198-205.

Kafourou V, Tong HJ, Day P, Houghton N, Spencer RJ, Duggal M. 2017. Outcomes and prognostic factors that influence the success of tooth autotransplantation in children and adolescents. Dent Traumatol. 33(5):393-399.

Kallu R, Vinckier F, Politis C, Mwalili S, Willems G. 2005. Tooth transplantations: a descriptive retrospective study. Int J Oral Maxillofac Surg. 34(7):745-755.

Kanavakis G, Ludwig B, Rosa M, Zachrisson B, Hourfar J. 2014. Clinical outcomes of cases with missing lateral incisors treated with the ' $\mathrm{t}$ '-mesialslider. J Orthod. 41(Suppl 1):S33-S38.

Keightley AJ, Cross DL, McKerlie RA, Brocklebank L. 2010. Autotransplantation of an immature premolar, with the aid of cone beam CT and computer-aided prototyping: a case report. Dent Traumatol. 26(2):195-199.

Lambrichts I, Driesen RB, Dillen Y, Gervois P, Ratajczak J, Vangansewinkel T, Wolfs E, Bronckaers A, Hilkens P. 2017. Dental pulp stem cells: their potential in reinnervation and angiogenesis by using scaffolds. J Endod. 43(9S):S12-S16.

Loubele M, Bogaerts R, Van DE, Pauwels R, Vanheusden S, Suetens P, Marchal G, Sanderink G, Jacobs R. 2009. Comparison between effective radiation dose of CBCT and MSCT scanners for dentomaxillofacial applications. Eur J Radiol. 71(3):461-468.

Maes F, Collignon A, Vandermeulen D, Marchal G, Suetens P. 1997. Multimodality image registration by maximization of mutual information. IEEE Trans Med Imaging. 16(2):187-198.

Meschi N, EzEldeen M, Torres Garcia AE, Jacobs R, Lambrechts P. 2018. A retrospective case series in regenerative endodontics: trend analysis based on clinical evaluation and 2- and 3-dimensional radiology. J Endod. 44(10): 1517-1525.

Obregon F, Vaquette C, Ivanovski S, Hutmacher DW, Bertassoni LE. 2015. Three-dimensional bioprinting for regenerative dentistry and craniofacial tissue engineering. J Dent Res. 94(9, Suppl):143S-152S.

Paulsen HU, Andreasen JO, Schwartz O. 2006. Tooth loss treatment in the anterior region: autotransplantation of premolars and cryopreservation. World J Orthod. 7(1):27-34.

Petti S, Glendor U, Andersson L. 2018. World traumatic dental injury prevalence and incidence, a meta-analysis-one billion living people have had traumatic dental injuries. Dent Traumatol. 34(2):71-86.

Shahbazian M, Jacobs R, Wyatt J, Denys D, Lambrichts I, Vinckier F, Willems G. 2013. Validation of the cone beam computed tomography-based stereolithographic surgical guide aiding autotransplantation of teeth: clinical case-control study. Oral Surg Oral Med Oral Pathol Oral Radiol. 115(5):667-675.

Shahbazian M, Jacobs R, Wyatt J, Willems G, Pattijn V, Dhoore E, Van Lierde C, Vinckier F. 2010. Accuracy and surgical feasibility of a CBCT-based stereolithographic surgical guide aiding autotransplantation of teeth: in vitro validation. J Oral Rehabil. 37(11):854-859.

Sharma AB, Vargervik K. 2006. Using implants for the growing child. J Calif Dent Assoc. 34(9):719-724

Smith EE, Angstadt S, Monteiro N, Zhang W, Khademhosseini A, Yelick PC. 2018. Bioengineered tooth buds exhibit features of natural tooth buds. J Dent Res. 97(10):1144-1151.

Tsukiboshi M. 2002. Autotransplantation of teeth: requirements for predictable success. Dent Traumatol. 18(4):157-180.

Wei F, Song T, Ding G, Xu J, Liu Y, Liu D, Fan Z, Zhang C, Shi S, Wang S. 2013. Functional tooth restoration by allogeneic mesenchymal stem cell-based bio-root regeneration in swine. Stem Cells Dev. 22(12):17521762.

Zachrisson BU, Stenvik A, Haanaes HR. 2004. Management of missing maxillary anterior teeth with emphasis on autotransplantation. Am J Orthod Dentofacial Orthop. 126(3):284-288. 